\title{
Traumatic Dislocation of the Posterior Tibial Tendon: A Case Report and Review of the Literature
}

\author{
Hector Masaragian ${ }^{1}$, Ezequiel Luege ${ }^{2}$, Fernando Perin ${ }^{3}$, Hernan Coria $^{4}$, Luciano Mizdraji ${ }^{5}$, Juan M Santos ${ }^{6}$, Leonel Rega ${ }^{7}$,
} Nicolas Ameriso ${ }^{8}$

\begin{abstract}
Background: Traumatic dislocation of the posterior tibial tendon (PTT) is a highly unusual pathology. Diagnosis is based on suspicion and clinical examination.

Case description: A 35-year-old male patient suffered a car accident and presented swelling, pain, and instability in the medial side of the right ankle and ecchymosis, on the affected side, associated with deltoid ligament injury. The mechanisms of the injury have not been completely defined but, in most cases, it has been associated with forced dorsiflexion and inversion of the ankle. He was surgically treated 2 weeks after the procedure. Conclusion: Surgical treatment is mandatory. The goal is to relocate the tendon in its normal anatomical position and recreate a competent flexor retinaculum.

Clinical significance: We present an unusual case report with good clinical results after surgical treatment and a surgical tip not described before. Keywords: Case report, Dislocation, Posterior tibial tendon, Traumatic.

Journal of Foot and Ankle Surgery (Asia Pacific) (2019): 10.5005/jp-journals-10040-1106
\end{abstract}

\section{BACKGROUND}

Traumatic dislocation of the posterior tibial tendon (PTT) is a highly unusual pathology. Very few cases have been reported in the literature.

The first case was reported by Martins in 1874, who described an injury in himself after falling from a hot air balloon.

According to several authors, typical presentation is pain and instability after trauma, usually an inversion of the ankle, during sports practice. It may be misdiagnosed in many cases.

Diagnosis is based on suspicion and clinical examination. It is complemented with simple $\mathrm{X}$-rays and ankle magnetic resonance image (MRI) to rule out associated pathology. Surgical treatment is mandatory. ${ }^{1,2}$

\section{Case Description}

A 35-year-old male patient suffered a car accident with a motorcycle and had no history of previous trauma.

He was evaluated a week after the initial trauma, presenting difficulty to bear weight normally with swelling, pain, medial ankle instability, and ecchymosis. We observed the associated deltoid ligament injury that was treated after a week; he was unable to bear weight normally and presented swelling, pain, and instability in the medial side of the right ankle, and ecchymosis, on the affected side, associated with deltoid ligament injury.

Anterior to the medial malleolus, a structure compatible with the PTT was detected (Fig. 1).

An avulsive image in the area of insertion of the flexor retinaculum in the medial malleolus was found in plain X-rays (Fig. 2). The MRI also revealed a dislocation of the PTT with a complete damage of the superficial and deep deltoid ligament (Figs 3 to 5).

Surgical treatment was performed 2 weeks after the injury.

\section{Procedure}

The procedure was done under spinal anesthesia with a popliteal block (for postoperative analgesia) with tourniquet in the thigh.

\begin{abstract}
${ }^{1-8}$ Department of Foot and Ankle, Dr Masaragian-Cirugiadelpie.net, Autonomous City of Buenos Aires, Argentina

Corresponding Author: Hector Masaragian, Department of Foot and Ankle, Dr Masaragian-Cirugiadelpie.net, Autonomous City of Buenos Aires, Argentina, Phone: +54 1152494685, e-mail: masa@cirugiadelpie. net
\end{abstract}

How to cite this article: Masaragian $\mathrm{H}$, Luege $\mathrm{E}$, Perin $\mathrm{F}$, et al. Traumatic Dislocation of the Posterior Tibial Tendon: A Case Report and Review of the Literature. J Foot Ankle Surg (Asia Pacific) 2019;6(2):41-45.

Source of support: Nil

Conflict of interest: None

Contralateral hip elevation was placed to allow greater external rotation of the foot. A curvilinear retromalleolar incision was made up to the navicular. The flexor retinaculum was found completely torn, and the PTT was dislocated, anterior to the tibial malleolus. The tendon was irreducible manually without the release of the soft tissues. Tendon damage was not observed. As an intraoperative finding, the retromalleolar groove was flatter than usual, so a groove deepening was performed, with repair of the superficial and deep bundles of the deltoid ligament and the flexor retinaculum (Fig. 6). An augmentation with InternalBrace ${ }^{\mathrm{TM}}$ (Arthrex) was also performed to increase medial stability (Fig. 6).

\section{Postoperative Management}

Immobilization was applied for 3 weeks with the ankle at $90^{\circ}$ and slight inversion of the foot. At 3 weeks, stitches were removed, and a walker boot was placed until the 6 th week. Range of motion (ROM) exercises and gradual weight-bearing as tolerated were indicated associated with physical therapy.

A year after surgery, the patient presents no pain and mild residual edema. Clinically, he can stand in tiptoes, with good 

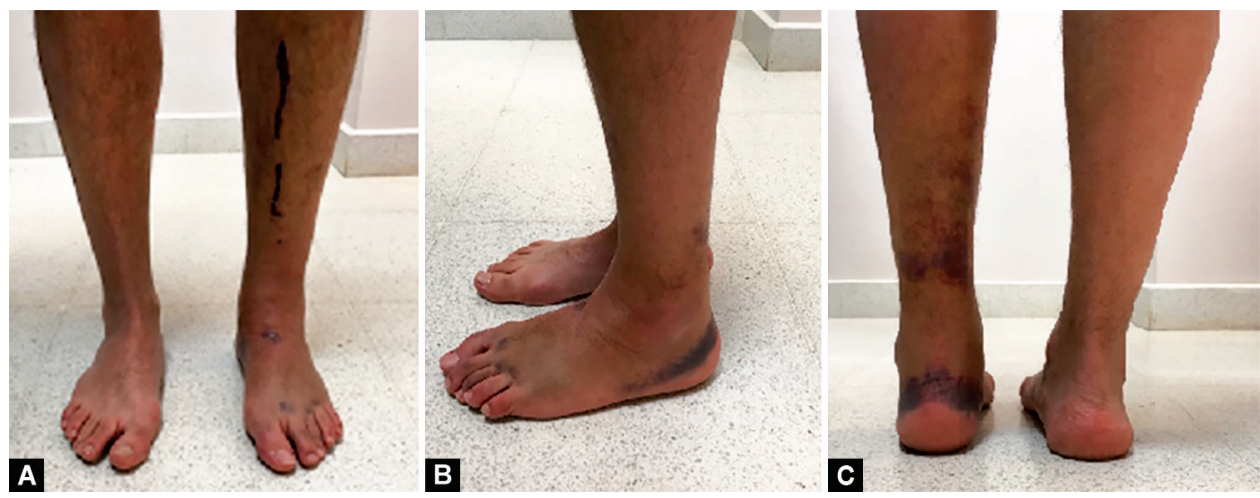

Figs $1 \mathrm{~A}$ to $\mathrm{C}$ : Clinical picture of the patient after the accident
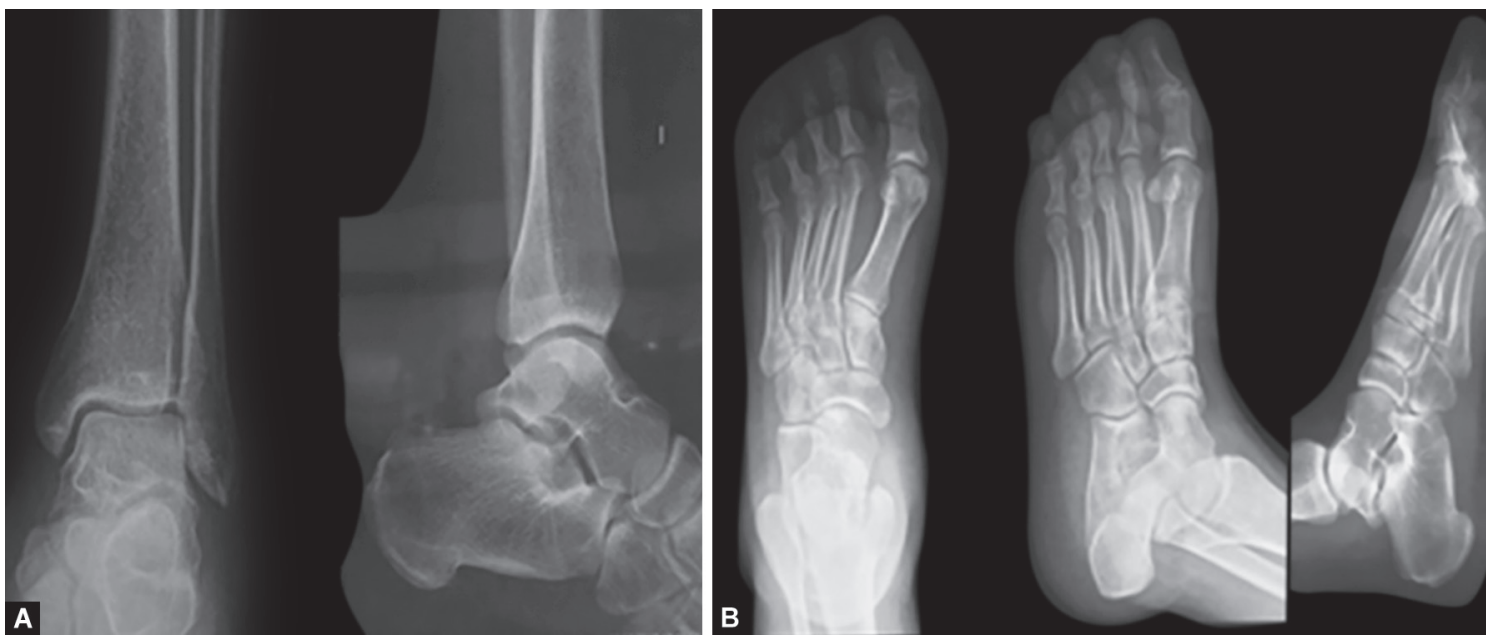

Figs $2 \mathrm{~A}$ and B: Radiographic presentation. (A) Front and lateral view of the ankle; (B) Front, oblique, and lateral view of the foot

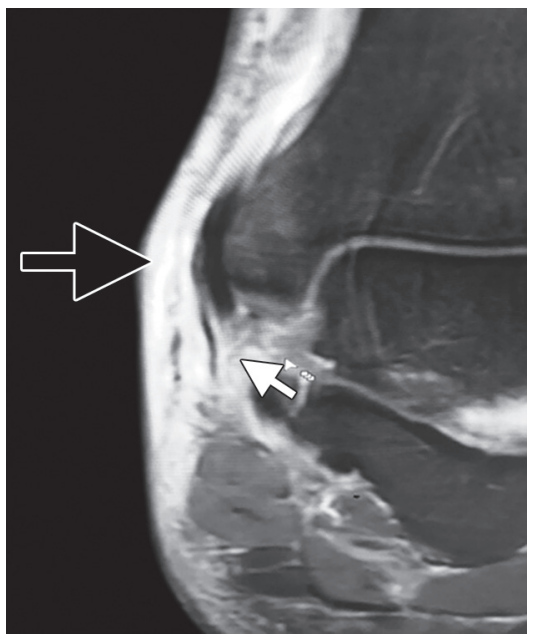

Fig. 3: MRI. In the thicker arrow, dislocation of the posterior tibial bone is seen, and in the thinnest arrow it indicates the deltoid lesion

elevation and inversion of the heel (Fig. 7). In control MRI, PTT muscle is slightly anterior subluxated but stable (Fig. 8).

\section{Discussion}

Lohrer and Nauck reported in a review of this pathology 61 published cases up to that moment. Only $14 \%$ of the patients (eight cases) presented an avulsion fracture.
The mechanisms of the injury have not been completely defined but, in most cases, they have been associated with forced dorsiflexion and inversion of the ankle. Perlman et al. reported that the most probable mechanism of injury is a forced dorsiflexion of the foot combined with supination of the hindfoot and external rotation of the leg.

In physical examination, patients with acute dislocation present edema and ecchymosis on the medial malleolus, which makes immediate diagnosis difficult. When the edema decreases, a palpable mobile cord can be observed running obliquely on the medial aspect of the ankle. Patients often report pain in the medial region, inability to bear weight in the forefoot, difficulty on stairs, and a feeling of ankle block. ${ }^{3-6}$

In a review article published by Jeong et al., $53.1 \%$ of cases were initially misdiagnosed, and an average of 4.4 months passed until tendon dislocation was diagnosed. They described three types of injuries (Table 1). ${ }^{2}$

Strydom presented a new classification with subtypes (Table 2). ${ }^{7}$ On surgical exploration, the findings include a rupture or avulsion of the flexor retinaculum, a flat retro malleolar sulcus, elevation of the retinaculum, or a loose retinaculum., ${ }^{1,8}$

Rolf et al. reported a case of dislocation of the PTT that was diagnosed by $\mathrm{CT}$ and ultrasound. In this study, the retromalleolar groove architecture was not mentioned. ${ }^{9}$

Soler et al. studied the anatomical variations of the depth and width of the medial retromalleolar groove in 25 cadaver dissections measured in plaster casts. The dimensions of the groove showed 


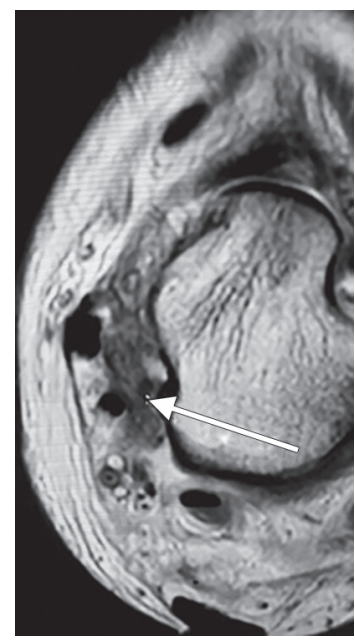

Fig. 4: MRI, axial view. Calcaneonavicular ligament without signs of injury (arrow)

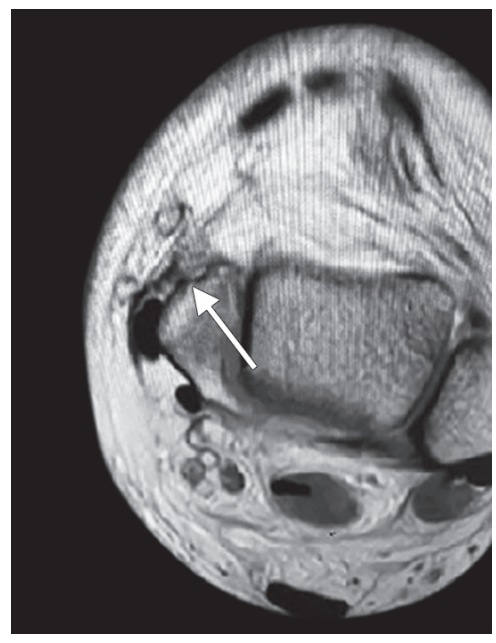

Fig. 5: MRI of the flexor retinaculum lesion (arrow)
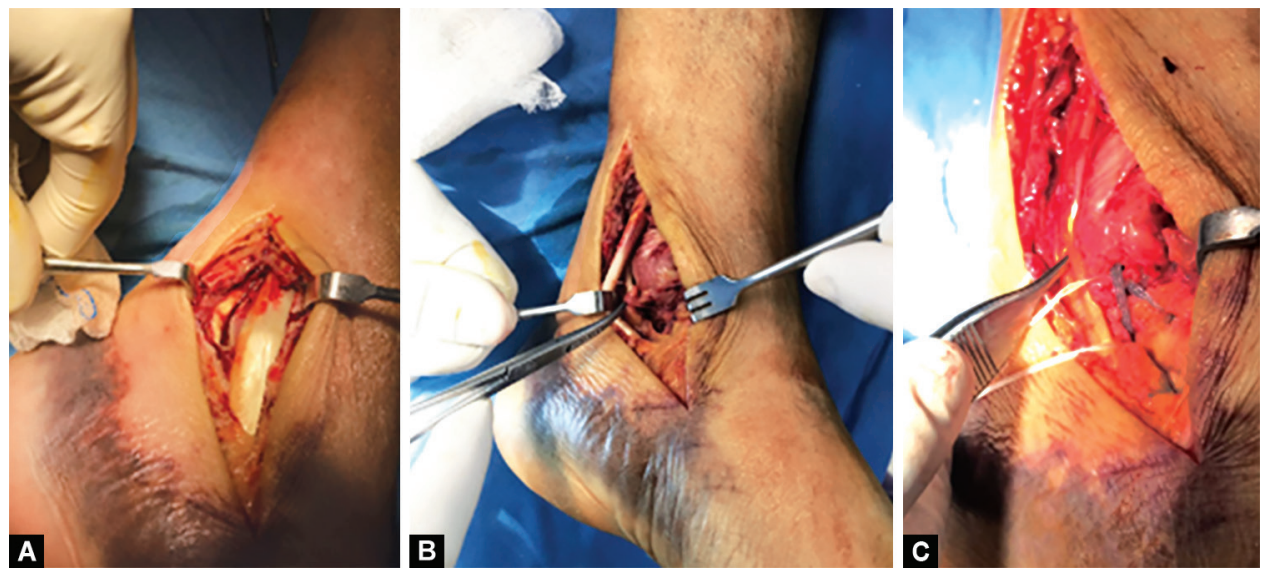

Figs $6 \mathrm{~A}$ to C: Intraoperative image: (A) Tendon dislocated anterior to the medial malleolus; $(\mathrm{B})$ Reduction of the injured tendon and the deltoid ligament; (C) Tendon in the retromalleolar region with the InternalBrace ${ }^{\mathrm{TM}}$ implanted

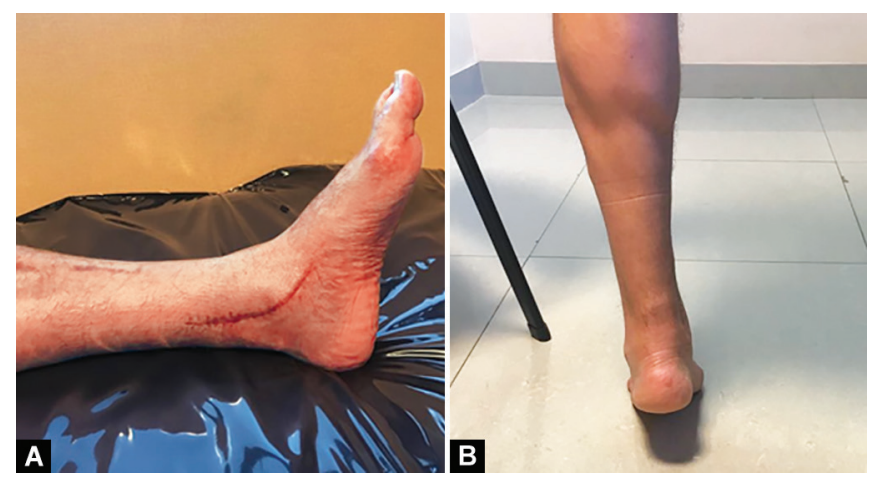

Figs $7 \mathrm{~A}$ and B: One-year postoperative clinical examination. Patient can stand with the injured foot with good heel rise

a high degree of variability, with a width from 6 to $15 \mathrm{~mm}$ and a depth from 1.5 to $4 \mathrm{~mm}$. In this study, they also mention the use of $\mathrm{CT}$ to perform the reconstruction and measurement of the internal retromalleolar sulcus. ${ }^{3,9}$

Ouzanian and Myerson in 1992 have reported the largest clinical and surgical series with seven cases. They presented six
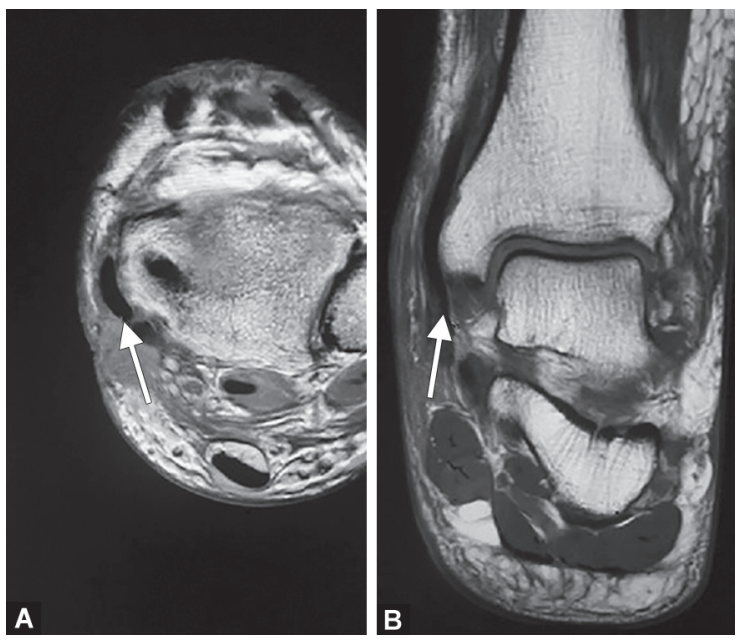

Figs $8 \mathrm{~A}$ and $\mathrm{B}$ : Postoperative MRI image: $(\mathrm{A})$ Axial view showing the subluxated tendon (arrow); (B) Coronal view showing the repaired deltoid ligament (arrow) 


\begin{tabular}{ll} 
Table 1: Classification proposed by Jeong et al. & \\
\hline Type & Injury \\
\hline I & $\begin{array}{l}\text { Subcutaneous dislocation due to rupture of the anterior } \\
\text { part of the flexor retinaculum }\end{array}$ \\
II & $\begin{array}{l}\text { Subperiosteal subluxation due to the disinsertion of the } \\
\text { anterior portion of the flexor retinaculum with a flap } \\
\text { periosteal remnant where the posterior tibial remains } \\
\text { lodged }\end{array}$ \\
& Avulsion fracture of the flexor retinaculum
\end{tabular}

Table 2: Classification proposed by Strydom

\begin{tabular}{ll}
\hline Type $\quad$ Subtype & Injury \\
\hline I & Avulsion injury (the most common) \\
& similar to the classification of \\
& dislocation of the peroneal tendons of \\
& Eckert and Davies \\
& Pure avulsion of the flexor \\
& retinaculum of the medial malleolus \\
& Avulsion of the flexor retinaculum \\
& with bone fragment of the medial \\
& malleolus \\
& Retinal tear (associated with higher \\
& energy injuries) \\
& Hypotrophic or deficient retinaculum, \\
& this pathological feature can occur in \\
& Cases with a long diagnostic delay, \\
& medical comorbidities with soft tissue \\
& Complications and iatrogenic \\
III & retinaculum injury flexor \\
&
\end{tabular}

traumatic cases and one that occurred after multiple cortisone injections over a period of 18 months. The average time to diagnosis was 9 months. The patients were subjected to various conservative treatments without success. In this study, only two patients had positive findings on MRI of a PTT dislocation. In three of the seven patients, surgical exploration revealed tenosynovitis of the posterior tibialis and an avulsion lesion of the retinaculum. The malleolar retro groove was shallow in four of the seven cases. The surgical treatment consisted of the repair of the retinaculum in four cases and recreation of the retinaculum using local tissue in three cases. Two patients had a groove-deepening procedure. In the final follow-up, five patients were asymptomatic, one was gradually improving with time, and only one had chronic residual pain. ${ }^{9-11}$

Bencardino et al. reported seven cases of dislocation of the PTT in MRI. The mechanism of injury was acute dorsiflexion of the ankle on two occasions and major trauma in five. There were three tibial malleolar fractures. In one patient, the diagnosis was made before the complementary examination. The MRI showed a dislocated tendon in five patients and a subluxation in two. In one case, a partial rupture of the tendon was observed. The retromalleolar sulcus was superficial in one, oblique in another, and normal in five. In this series, a rupture of the retinaculum was observed on two occasions and avulsion of the same in five patients. Only two underwent surgery for dislocation of the tendon. The authors concluded that MRI was a valuable tool for diagnosis and surgical planning., 9
Prato et al. described the value of ultrasound in the acute phase. ${ }^{13}$ Gambhir et al. reported a late diagnosis of this pathology, with the formation of a bone spur in the medial malleolus at the avulsion site of the flexor retinaculum. ${ }^{11,13}$

Larsen and Lauridsen reported the traumatic dislocation of the PTT in an 18-year-old man and a 36-year-old woman. Both patients appeared to have suffered dorsiflexion and inversion of the affected foot. In the surgery, they found the flexor retinaculum torn and repaired it in a primary form with immobilization for 6 weeks, obtaining excellent results. ${ }^{6,14}$

Biedert described a case of an 18-year-old male patient who suffered a traumatic PTT dislocation. In surgery, the flexor retinaculum was completely reconstructed using only suture, with anchoring through holes in the medial malleolus. The author suggested strengthening the retinaculum with local tissue. The results were reported as excellent. ${ }^{4,15}$

\section{Conclusion}

Currently there is no consensus in the literature on the best method of treatment, but all authors agree that surgical open procedure is mandatory. ${ }^{9}$

In the series of patients treated nonsurgically, there is no mention of the kind of method used, long-term results, or sequelae.

The goal of the surgery is to relocate the tendon in its normal anatomical position and recreate a competent flexor retinaculum. In some cases, medial groove deepening is necessary.

We found no long-term MRI evaluations of results concerning the PTT position after surgery and the relation with clinical outcomes.

The fact of presenting only one case does not allow us to conclude that the result of the treatment was the best option, even with our patient satisfaction.

In the mentioned series, injuries of the deltoid ligament are not described, as we found in our patient, nor the augmentation with InternalBrace ${ }^{\mathrm{TM}}$ to increase medial stability.

\section{References}

1. Godino M, Vides M, Guerado E. Traumatic dislocation of posterior tibial tendon by avulsion of flexor retinacular release. Reconstruction with suture anchors. Rev Esp Cir Ortop Traumatol 2015;59(3):211-214. DOI: 10.1016/j.recote.2015.03.002.

2. Jeong S, Hwang S, Kim D, et al. A new surgical technique for traumatic dislocation of posterior tibial tendon with avulsion fracture of medial malleolus. Acta Orthop Traumatol Turc 2015;49(6):690-693. DOI: 10.3944/AOTT.2015.15.0021.

3. Bencardino J, Beltran J, Broker M, et al. MR imaging of dislocation of the posterior tibial tendon. AJR Am J Roentgenol 1997;169(4): 1109-1112. DOI: 10.2214/ajr.169.4.9308473.

4. Nuccion SL, Hunter DM, DiFiori J. Dislocation of the posterior tibial tendon without disruption of the flexor retinaculum: a case report and review of the literature. Am J Sports Med 2001;29(5):656-659. DOI: 10.1177/03635465010290052101.

5. Lohrer H, Nauck T. Posterior tibial tendon dislocation: a systematic review of the literature and presentation of a case. Br J Sports Med 2010;44(6):398-406. DOI: 10.1136/bjsm.2007.040204.

6. Gambardella GV, Donegan R, Caminear DS. Isolated dislocation of the posterior tibial tendon in an amateur snowboarder: a case report. J Foot Ankle Surg 2014;53(2):203-207. DOI: 10.1053/j.jfas.2013.10.006.

7. Strydom A, Saragas NP, Tladi M, et al. Tibialis posterior tendon dislocation: a review and suggested classification. J Foot 
Ankle Surg 2017;56(3):656-665. DOI: 10.1053/j.jfas.2017.01. 006.

8. Mitchell K, Mencia MM, Hoford R. The foot tibialis posterior tendon dislocation: a case report. Foot (Edinb) 2011;21(3):154-156. DOI: 10.1016/j.foot.2010.10.005.

9. Goucher NR, Coughlin MJ, Kristensen RM. Dislocation of the posterior tibial tendon: a literature review and presentation of two cases. lowa Orthop J 2006;26:122-126.

10. Kim SC, Kim SH, Kim SJ. A case report: large trichobezoar causing rapunzel syndrome. Medicine (Baltimore) 2016;95(22):e3745. DOI: 10.1097/MD.0000000000003745.

11. Ouzounian TJ, Myerson MS. Dislocation of the posterior tibial tendon. Foot Ankle 1992;13(4):215-219. DOI: 10.1177/ 107110079201300409.
12. Trividi $M$, Brown $E$, Lese $A$, et al. Lateral dislocation and incarceration of the posterior tibial tendon through the distal tibiofibular syndesmosis. Skeletal Radiol 2014;43(8):1175-1178. DOI: 10.1007/ s00256-014-1859-9.

13. Aguiar ROC, Cabral MVG, Moura BB, et al. Dislocation of the flexor digitorum longus and posterior tibial tendons without fracture dislocation of the ankle: a case report. Foot Ankle Int 2007;28(11): 1187-1189. DOI: 10.3113/FAI.2007.1187.

14. Ikuta Y, Adachi N, Nakasa T, et al. Postoperative repeat dislocation of the posterior tibial tendon: a case report. J Foot Ankle Surg 2017;56(3):687-691. DOI: 10.1053/j.jfas.2017.01.045.

15. Healy WA, Starkweather KD, Gruber MA. Chronic dislocation of the posterior tibial tendon: a case report. Am J Sports Med 1995;23(6):776-777. DOI: 10.1177/036354659502300625. 